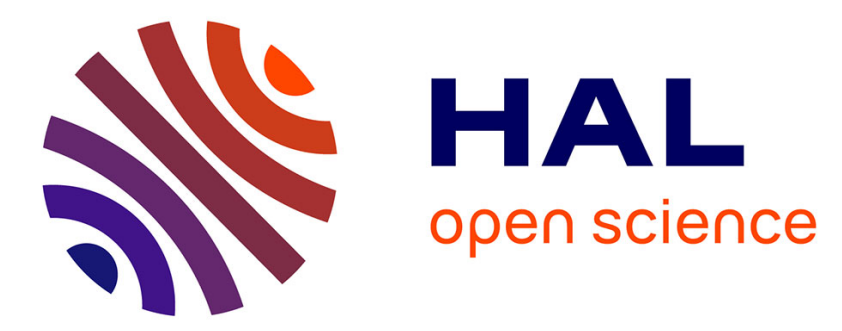

\title{
Predictable surface ablation of dielectrics with few-cycle laser pulse even beyond air ionization
}

\author{
Corinne Pasquier, M. Sentis, O. Uteza, N. Sanner
}

\section{To cite this version:}

Corinne Pasquier, M. Sentis, O. Uteza, N. Sanner. Predictable surface ablation of dielectrics with few-cycle laser pulse even beyond air ionization. Applied Physics Letters, 2016, 109 (5), pp.051102. 10.1063/1.4960152 . hal-01630866

\section{HAL Id: hal-01630866 https://hal.science/hal-01630866}

Submitted on 8 Nov 2017

HAL is a multi-disciplinary open access archive for the deposit and dissemination of scientific research documents, whether they are published or not. The documents may come from teaching and research institutions in France or abroad, or from public or private research centers.
L'archive ouverte pluridisciplinaire HAL, est destinée au dépôt et à la diffusion de documents scientifiques de niveau recherche, publiés ou non, émanant des établissements d'enseignement et de recherche français ou étrangers, des laboratoires publics ou privés. 


\section{AIP Apoled Phosics \\ Letters}

Predictable surface ablation of dielectrics with few-cycle laser pulse even beyond air ionization

C. Pasquier, M. Sentis, O. Utéza, and N. Sanner

Citation: Applied Physics Letters 109, 051102 (2016); doi: 10.1063/1.4960152

View online: http://dx.doi.org/10.1063/1.4960152

View Table of Contents: http://scitation.aip.org/content/aip/journal/apl/109/5?ver=pdfcov

Published by the AIP Publishing

\section{Articles you may be interested in}

Atomistic simulation of laser-pulse surface modification: Predictions of models with various length and time scales

J. Appl. Phys. 117, 135901 (2015); 10.1063/1.4916600

Submicron surface patterning by laser ablation with short UV pulses using a proximity phase mask setup J. Appl. Phys. 107, 063106 (2010); 10.1063/1.3331409

Toward determinism in surface damaging of dielectrics using few-cycle laser pulses

Appl. Phys. Lett. 96, 071111 (2010); 10.1063/1.3309700

Ablative modification of graphite surfaces by single intense femtosecond pulses

J. Appl. Phys. 100, 036103 (2006); 10.1063/1.2219376

On the angular dependence of focused laser ablation by nanosecond pulses in solgel and polymer materials Appl. Phys. Lett. 84, 1680 (2004); 10.1063/1.1655702

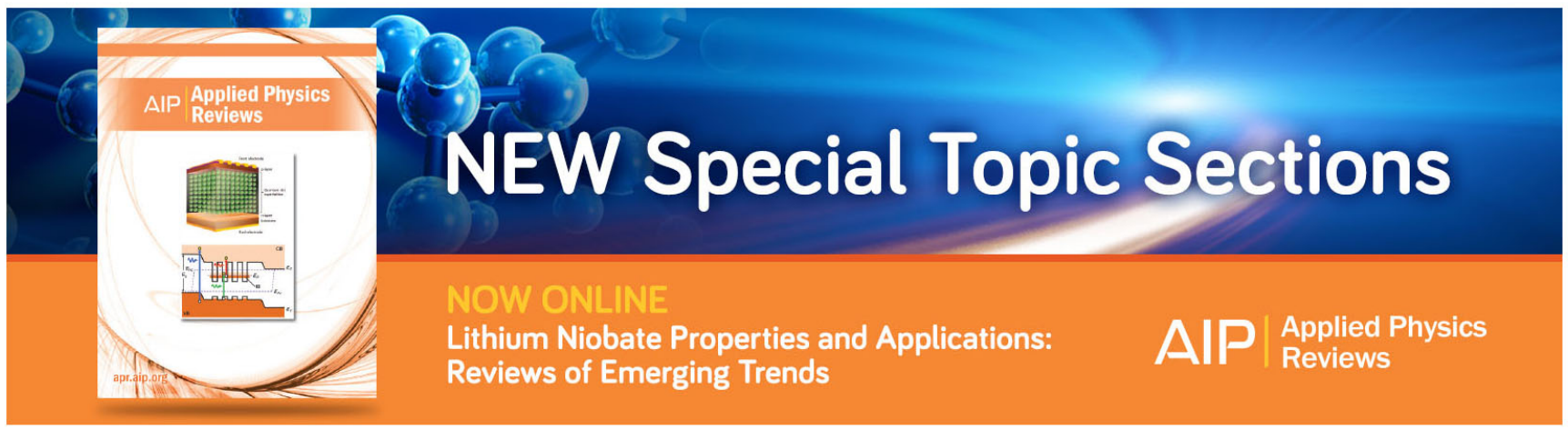




\title{
Predictable surface ablation of dielectrics with few-cycle laser pulse even beyond air ionization
}

\author{
C. Pasquier, ${ }^{\text {a) }}$ M. Sentis, O. Utéza, and N. Sanner ${ }^{\text {b) }}$ \\ Aix Marseille Univ, CNRS, LP3, UMR7341, 13288 Marseille, France
}

(Received 10 June 2016; accepted 19 July 2016; published online 1 August 2016)

\begin{abstract}
We study surface ablation of dielectrics with single-shot few-cycle optical pulse ( $\sim 10 \mathrm{fs})$ in air, at intensities below and above the onset of air ionization. We perform 3D analysis and careful calibration of the fluence distribution at the laser focus, spanning from linear- to nonlinear- focusing regimes, enabling to thoroughly characterize the severe limitation of the fluence delivered onto the sample surface upon increase of incident pulse energy. Despite significant beam reshaping taking place at high fluence, we demonstrate that it is nevertheless possible to confidently predict the resulting crater profiles on fused silica surface, even in the regime of filamentation. Published by AIP Publishing. [http://dx.doi.org/10.1063/1.4960152]
\end{abstract}

Few-cycle laser pulses offer remarkable capabilities for laser-matter interaction, especially for ablation of transparent dielectric materials. Since the first experiments using $\sim 10$ fs pulses ${ }^{1,2}$ knowledge has progressed to highlight the most interesting features compared to longer femtosecond pulses: triggering of preferential nonlinear absorption mechanisms, leading to reinforcement of the deterministic character of interaction $^{3}$ as well as the need for only a low amount of energy to overpass material threshold. ${ }^{4}$ These two peculiarities confer the capability to machine matter with high resolution (below the diffraction limit) resulting in calibrated outcomes and minimal collateral effects and affected zone.

However, focusing ultrashort laser pulses in air implies natural limitations to linear beam propagation prior the target. When the peak power of a Gaussian beam exceeds the critical power $\mathrm{P}_{\mathrm{cr}}$ (around $3 \mathrm{GW}$ in air at $800 \mathrm{~nm}$ (Ref. 5)), self-focusing related to the modulation of the refractive index by optical Kerr effect causes the beam to collapse before the geometrical (linear) focus. ${ }^{5,6}$ This provokes an increase in the intensity, yielding photoionization of air (at intensities of few $10^{13} \mathrm{~W} / \mathrm{cm}^{2}$ ), and creation of plasma whose free electrons contribute negatively to the refraction index and defocus the beam. Laser filaments can also form as a result of the dynamic balance between these two competing effects; ${ }^{5}$ they have been extensively studied for low numerical aperture, typically NA $\sim 10^{-2}-10^{-3}$ (see, for example, the review of Ref. 7). However, micrometric-scale surface ablation of dielectric materials requires stronger external focusing and pulse energies above the threshold for nonlinear propagation effects in air. Very few experimental studies have examined femtosecond pulse propagation in air with tightly focused beams. ${ }^{8-11}$ In previous works done in such conditions, ${ }^{12}$ we determined an intensity, equal to $\mathrm{I}_{\mathrm{NL}}$ $=1.4 \times 10^{14} \mathrm{~W} / \mathrm{cm}^{2}$, above which the beam undergoes distortions due to the nonlinear effects taking place in air. Nevertheless, the consequences on ablation outcomes (as for instance the diameter of the ablated crater) using few-cycle pulses have not been investigated so far.

\footnotetext{
a) pasquier@1p3.univ-mrs.fr

b)sanner@1p3.univ-mrs.fr
}

In this letter, we show that, despite pre-focal nonlinear propagation and air ionization that yield severe beam distortions, prediction and realization of high-quality ablation crater are still attainable in air. By carefully measuring the 3D beam distribution, we highlight the need to re-define the fluence at the position of the sample surface, and we reveal and characterize its limitation upon increase of the incident pulse energy. We illustrate the interest of this knowledge by demonstrating that the range of applicability of few-cycle pulses to ablation in air ambiance is thereby extended far beyond the natural limit which corresponds to the appearance of nonlinear effects in air.

The experimental setup using ASUR platform has been described in detail elsewhere. ${ }^{12}$ It relies on a Ti:Sa laser system delivering linearly polarized $25 \mathrm{fs}, 1 \mathrm{~mJ}$ pulses at $100 \mathrm{~Hz}$, and $800 \mathrm{~nm}$ central wavelength. The spectrum is further enlarged up to $160 \mathrm{~nm}$ at $1 / \mathrm{e}^{2}(720-880 \mathrm{~nm})$ through crosspolarized wave generation in two cascaded $\mathrm{BaF}_{2}$ crystals. A set of chirped mirrors followed by two thin $\mathrm{SiO}_{2}$ wedges enable to accurately compress the pulse down to $12 \pm 1$ fs FWHM with a maximal energy $( \pm 1.6 \% \mathrm{rms})$ of $50 \mu \mathrm{J}$. The incident beam radius measured at $1 / \mathrm{e}^{2}$ with respect to intensity is $2.5 \mathrm{~mm}$. The beam is further focused with an off-axis parabolic mirror (gold-coated, $\mathrm{f}^{\prime}=50 \mathrm{~mm}$ ) on the target surface with normal incidence $(\mathrm{NA}=0.05)$. The pulse duration is measured with a second-order autocorrelator located just before the focusing mirror. The tested dielectric material is amorphous fused silica $\mathrm{SiO}_{2}$ (superpolished Suprasil from Heraeus, thickness $2.5 \mathrm{~mm}$ ) chosen for its wide range of applications in optics and material science. However, the results may apply to any other material. All the experiments are performed in single-pulse regime, i.e., every pulse irradiates a fresh zone of the sample. The ablated craters are quantitatively characterized by confocal microscopy.

We first characterize the beam distribution without the sample by longitudinal imaging, achieved by collecting the beam using a 0.14-NA microscope objective and imaging it onto a beam analyzer with a $14 \times$ magnification. We measure 2D transverse images of the beam in several planes in the vicinity of the focus, and we reconstruct the 3D intensity distribution of beam propagation for every incident energy [Fig. 1]. 
At low incident energy, we measure Gaussian beam distribution and propagation [Fig. 1(a)]. The position $z=0$ on the optical axis corresponds to the focal plane, referred to as "linear" focus, in the absence of nonlinear propagation in air. The fluence is defined by $F_{G}(r, z=0)=F_{0} \exp \left[-2 r^{2} / w_{0}^{2}\right]$ with $F_{0}=2 E / \pi w_{0}^{2}$ the peak fluence ( $E$ is the pulse energy) and $w_{0}=11.0 \mu \mathrm{m}$ the measured beam radius at $1 / \mathrm{e}^{2}$. The sample is then precisely located in the focal plane using combined energy-scan and z-scan procedures with an accuracy of $50 \mu \mathrm{m}$, much better than the Rayleigh range $\mathrm{z}_{\mathrm{R}}=\pi \mathrm{w}_{0}^{2} /$ $\mathrm{M}^{2} \lambda=396 \mu \mathrm{m}$ (with measured $M^{2}=1.2$ ).

For Gaussian beam, and assuming deterministic character of interaction, the diameter $D$ of the crater should satisfy the relationship ${ }^{13}$ (expressed either in terms of fluence or energy)

$$
D^{2}=2 w_{0}^{2} \ln \left(F_{0} / F_{t h}\right)=2 w_{0}^{2} \ln \left(E / E_{t h}\right) .
$$

The ablation threshold $F_{t h}$ is defined by $D^{2}=0$. Figure 2 shows that the experimental data are fully in accordance with this equation only for low incident energies, until $E_{N L}^{a i r}$ $=7.5 \mu \mathrm{J}$ (corresponding intensity $I_{N L}^{a i r}=1.65 \times 10^{14} \mathrm{~W} \mathrm{~cm}^{-2}$ ). As expected, this value is slightly above the intensity we previously determined, ${ }^{12}$ the small difference being related to the observable used for defining it. In our former experiment, we indeed measured the energy above which the beam focal plane begins to move out of the linear focus while here we observe the consequences of such displacement on an outcome of the interaction, e.g., the ablation diameter.

Importantly, this enables to retrieve the ablation threshold of the fused silica sample $\left(F_{t h}=1.8 \mathrm{~J} \mathrm{~cm}^{-2}\right.$, expressed in terms of peak fluence), which is well below the energy level corresponding to the onset of nonlinear effects. In addition, the

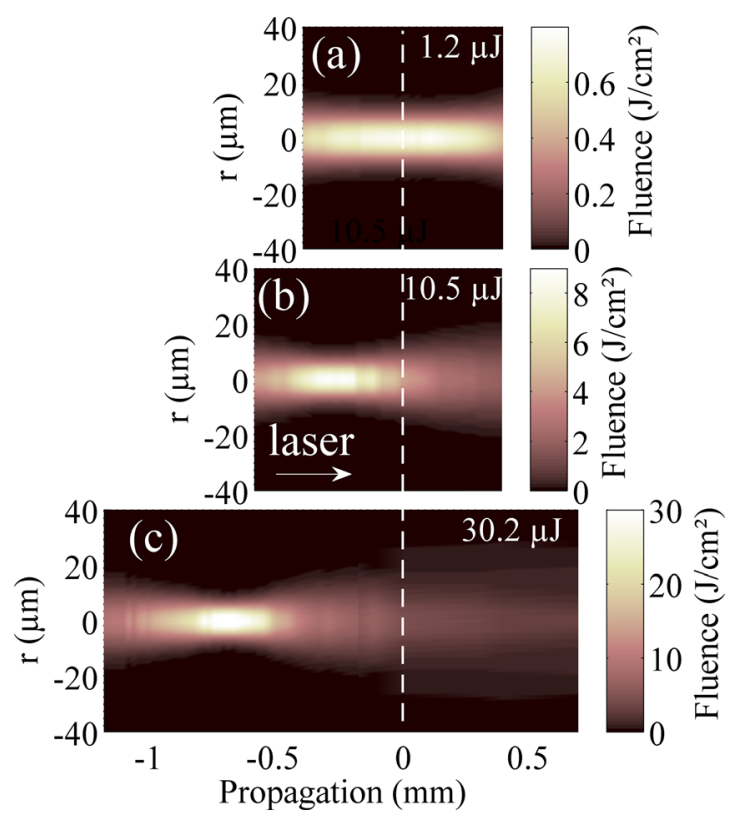

FIG. 1. 2D mapping of the reconstructed fluence along beam propagation for three different energies corresponding to the three regimes of propagation identified (see after). The fluence is calibrated by taking into account the energy conservation and the measured beam size, considering Gaussian beam propagation for cases (a) and (b) and superposition of Gaussian and superGaussian beams for case (c). The position $\mathrm{z}=0$ ("linear" focus) is indicated by the dashed line.

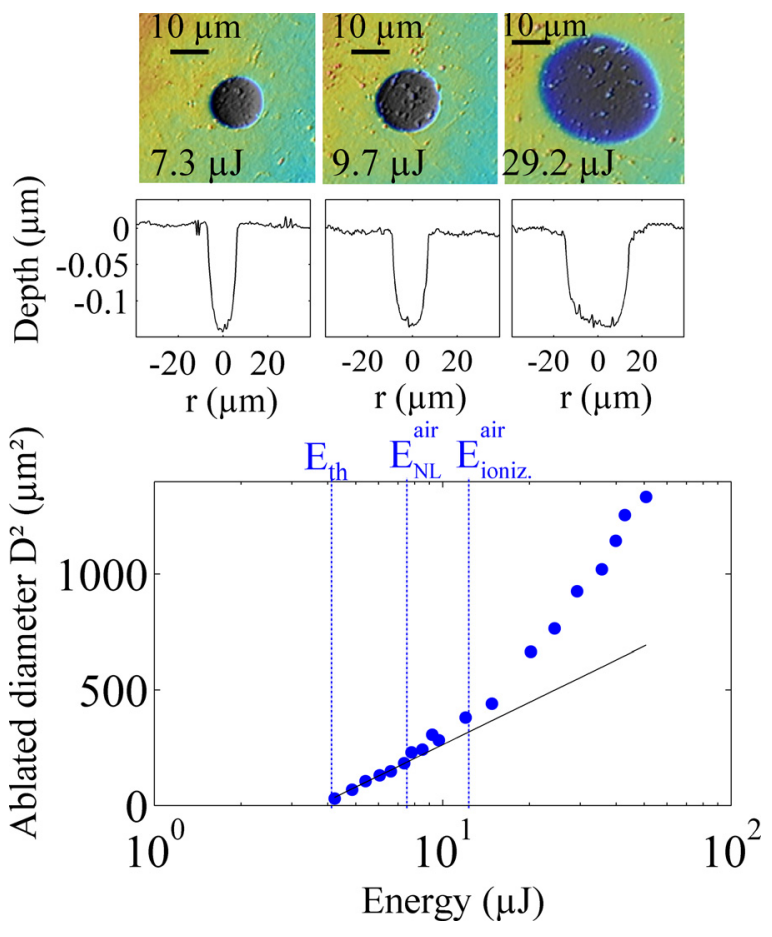

FIG. 2. Measured ablated diameters squared $\mathrm{D}^{2}$ as a function of incident energy (logarithmic scale). The numerical fit (continuous line) is obtained using the equation $D^{2}=2 w_{0}^{2} \ln \left(E / E_{t h}\right)$ for data corresponding to $E<E_{N L}^{a i r}$. The ablation threshold $\mathrm{E}_{\mathrm{th}}=3.6 \mu \mathrm{J}$ is obtained when the fit reaches $\mathrm{D}^{2}=0$. The data of ablated diameters are averaged over 10 points, using confocal microscopy. Three craters and 1-D profiles are shown in the three different regimes.

slope of the fit gives the beam waist, $w_{0}=11.3 \mu \mathrm{m}$, which is in excellent agreement with the measured value. Thus, up to $E=E_{N L}^{a i r}$ (corresponding to $F=4.1 \mathrm{~J} \mathrm{~cm}^{-2}=2.3 F_{t h}$ ), the crater diameter is completely and easily predictable. This is a finding of high practical importance: indeed, working under vacuum environment is not absolutely mandatory when using $\sim 10 \mathrm{fs}$ pulses, provided that the fluence is properly adjusted. A range of fluence close to threshold exists such that ablation in air is not hampered.

For incident energies above $E_{N L}^{a i r}$, crater diameters deviate from Eq. (1), with dimensions larger than expected. Beam analysis [Figs. 1(b) and 1(c)] indeed reveals how the nonlinear propagation in air changes the beam properties prior the linear focus. Note that for high energies the accuracy of the imaging system is somewhat lowered in the region up to the nonlinear focus only, because in this case the beam is measured through a medium (air) developing optical aberrations. However, in our experiment, the main interest is to know the details of the beam in the plane of the sample, thus in an observation zone where the imaging set-up is free of any optical aberrations. At this point, let us specify also that our aim is not to perform a dedicated study of beam propagation in air under external focusing, and not to characterize the density of the plasma generated in air but rather to link ablation results to beam analysis. In particular, self-focusing and air ionization cause changes in the z-position of the actual focus, as well as on further propagation properties. As a result, the actual beam at the sample surface (still located in the linear focus plane) is severely distorted, so that ablation may result in craters of completely different characteristics. The crucial point for prediction of crater diameter is the ability to accurately depict the 


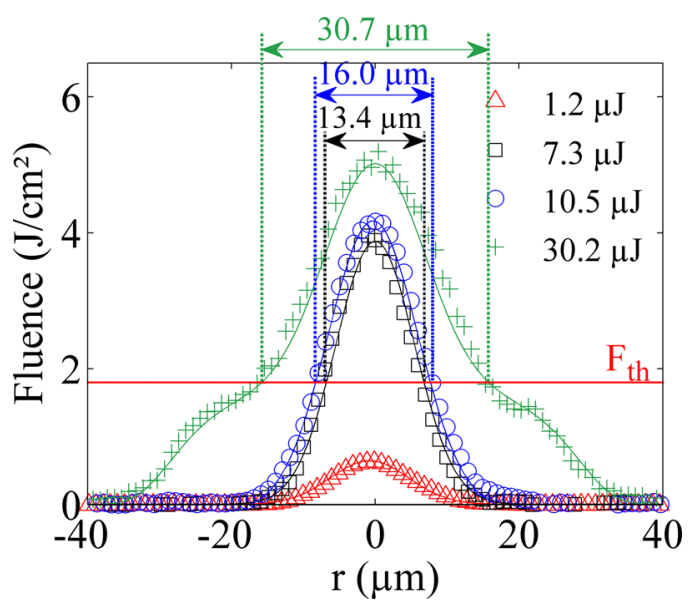

FIG. 3. Beam profile at the linear focus (i.e., at the surface of the sample) for three incident energies. Every profile is fitted by the equation $F(r)$ $=\sum_{n} E \alpha_{n}\left(\frac{2^{1 / n} n}{\pi w_{n}^{2} \Gamma(1 / n)}\right) \exp \left[-2\left(\frac{r}{w_{n}}\right)^{2 n}\right]$, where the order $\mathrm{n}$, the radius $w_{n}$ at $1 / \mathrm{e}^{2}$, and the weighting coefficient $\alpha_{n}$ are free parameters of the numerical fit for a given energy $E$. The threshold fluence $F_{t h}$ is also indicated, therefore, enabling to predict the crater diameter.

actual fluence $F(r)$ on the target surface, which we investigate now in detail.

Upon increase of the incident energy above $E_{N L}^{a i r}$, we identify two main regimes of beam propagation. The first regime (illustrated by Fig. 1(b)) corresponds to $E_{N L}^{\text {air }}<E$ $<E_{i o n i z}^{a i r}$. The upper energy limit of this regime was established from former experiments measuring the onset of blue shift and enlargement of the spectrum ${ }^{12}$ resulting from air ionization. Here, considering the impact on ablation diameters, it translates into $E_{\text {ioniz. }}^{\text {air }} \approx 12 \mu \mathrm{J}$ for which strong deviation from the Gaussian prediction is seen (see Fig. 2). In the regime $E_{N L}^{a i r}<E<E_{\text {ioniz. }}^{\text {air }}$, the beam profile is still Gaussianshaped but the focus is shifted towards the incoming laser beam direction (essentially due to self-focusing ${ }^{12}$ ) and its waist dimension $w_{0}^{\text {air }}$ is decreased compared to the one measured at linear focus. After this nonlinear focus, the beam diffracts and spreads transversely, further leading to larger dimension $w_{\text {sample }}^{\text {air }}$ on the sample surface (see Fig. 3, $\mathrm{E}=10.5 \mu \mathrm{J}$ ). Experimental data measured at energy levels representative of the different regimes are reported in Table I. A second regime is identified for energies $E \geq E_{\text {ioniz. }}^{\text {air }}$ (illustrated by Fig. 1(c)). In addition to the increased shift of the nonlinear focus prior the target, the beam intensity distribution is severely affected due to air ionization. Detrimentally for ablation experiments, the beam is no more Gaussian-shaped at the surface of the sample (see Fig. 3, $\mathrm{E}=30.2 \mu \mathrm{J})$.
In fact, such beam profile reproduces very well theoretical results from propagation in filamentary regime under tight external focusing. Indeed, Chin et al. ${ }^{14,15}$ have shown that in filamentary regime the intense laser beam is composed of single fundamental mode, the filament core, and all other higher order modes that form the background reservoir. ${ }^{16}$ The role and details of the energy reservoir were shown and clarified in Refs. 17-19. Following Ref. 17, the energy reservoir is expected to be (i) much larger than the core and (ii) to contain the major part of the pulse energy. Thus, the appearance of such beam distortions upon increase of incident energy highlights the need to re-define properly the fluence. With the aim of providing straightforward assessments and predictability of crater dimensions for ablation experiments, we now demonstrate that such fluence profiles can be well fitted by a combination of superGaussian functions (SG) of order $n$

$$
F(r)=\sum_{n} E \alpha_{n}\left(\frac{2^{1 / n} n}{\pi w_{n}^{2} \Gamma(1 / n)}\right) \exp \left[-2\left(\frac{r}{w_{n}}\right)^{2 n}\right] .
$$

The normalization factor (in bracket) depends on the order $n$ and $\Gamma(x)$ is the Euler Gamma function. For every beam that we measured for a given energy $\mathrm{E}$, the free parameters of the numerical fit are: the order $n$ of each participating SG function (note that $n=1$ corresponds to a Gaussian function), the radius $w_{n}$ at $1 / \mathrm{e}^{2}$, and the weighting coefficient $\alpha_{n}$ ensuring that the 2D integration over $r$ of the fluence function $F(r)$ equals the energy carried by the beam. Note that we neglect the small loss of energy due to plasma creation in air, since we measured in previous work ${ }^{12}$ that it is limited to $\sim 1 \%$, in agreement with recent numerical predictions ${ }^{20,21}$ in such sharp focusing conditions. In fact, the numerical fitting of the beam enables to evaluate the peak fluence value (used to calibrate Fig. 1), which is an essential data to know for predicting the crater diameter.

For illustrative purposes, we focus now on the case $\mathrm{E}=30.2 \mu \mathrm{J}$. Figure 3 shows an excellent agreement between the experimental beam profile on the sample and the numerical fit obtained from Eq. (2), corresponding to the superposition of a Gaussian function $(n=1)$ and two SG functions ( $n=3$ and 4$)$. The retrieved fitting parameters fully agree with Ref. 17: we obtain $w_{1}=14 \mu \mathrm{m}, w_{3}=31 \mu \mathrm{m}$, and $w_{4}=33 \mu \mathrm{m} ;$ and $\alpha_{1}=0.35, \alpha_{3}=0.60$, and $\alpha_{4}=0.05$, thus providing quantitative measurements of the respective dimensions and ratios of energy contained in the reservoir and in the main peak.

TABLE I. Linking beam and crater data. $\Delta z$ is the shift of location of the nonlinear focus prior the sample surface, $w_{0}^{\text {air }}$ is the corresponding radius associated to peak fluence $F_{0}^{\max }$, whereas $w_{\text {sample }}^{\text {air }}$ and $F_{0}^{\text {sample }}$ are real values at the sample surface. For the case $\mathrm{E}=30.2 \mu \mathrm{J}$, the three values indicated refer, respectively, to Gaussian $(\mathrm{n}=1)$ and two SuperGaussian $(\mathrm{n}=3$ and $\mathrm{n}=4)$ functions. $D_{\text {predicted }}$ is the beam diameter at local fluence equal to $F_{\text {th }}$, to be compared to $D_{\text {measured }}$ (averaged over 10 craters).

\begin{tabular}{lccccccc}
\hline \hline Energy $(\mu \mathrm{J})$ & $\Delta z(\mu \mathrm{m})$ & $w_{0}^{\text {air }}(\mu \mathrm{m})$ & $w_{\text {sample }}^{\text {air }}(\mu \mathrm{m})$ & $F_{0}^{\text {max }}\left(\mathrm{J} \mathrm{cm}^{-2}\right)$ & $F_{0}^{\text {sample }}\left(\mathrm{J} \mathrm{cm}^{-2}\right)$ & $D_{\text {predicted }}(\mu \mathrm{m})$ & $D_{\text {measured }}(\mu \mathrm{m})$ \\
\hline 1.2 & 0 & 11.0 & 11.0 & 0.8 & 0.8 & No crater \\
7.3 & 0 & 11.0 & 11.0 & 3.8 & 3.8 & 13.4 & $13.5 \pm 0.9$ \\
10.5 & -250 & 8.1 & 12.5 & 9 & 4.3 & 16.0 & $16.8 \pm 1^{\mathrm{a}}$ \\
30.2 & -700 & 7.1 & $14 / 31 / 33$ & 36 & 5 & 30.7 & $30.5 \pm 1.2^{\mathrm{a}}$ \\
\hline \hline
\end{tabular}

${ }^{\mathrm{a}}$ Measured at $\mathrm{E}=9.7 \pm 0.5 \mu \mathrm{J}$ and $29.2 \pm 1.4 \mu \mathrm{J}$, respectively. 


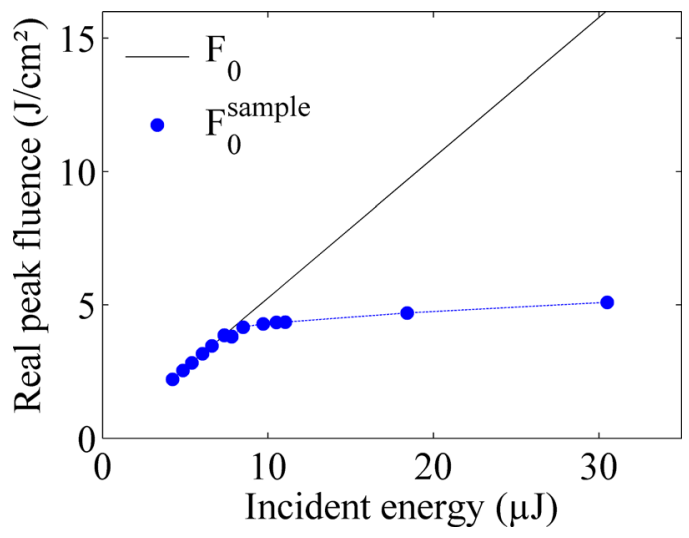

FIG. 4. Real peak fluence on the sample surface $F_{0}^{\text {sample }}$ as a function of incident pulse energy. The solid curve is the fluence $F_{0}=2 E / \pi w_{0}^{2}$ calculated with the parameters defined at low energy without nonlinear effects in air.

Finally, and thanks to the normalization factor in Eq. (2) issued from the fits for every case of energy investigated, the peak fluence gets calibrated and therefore so does the local fluence function $F(r)$ for every location $\mathrm{z}$ along propagation. Two important values are the peak fluence $F_{0}^{\max }$ attained at the nonlinear focus during propagation, and the real peak fluence at the sample surface $F_{0}^{\text {sample }}$. These values are also reported in Table I for three energy cases illustrating the three regimes identified. Now, considering our whole set of experimental data, Figure 4 plots the real peak fluence at the sample surface $F_{0}^{\text {sample }}$ upon increase of incident energy. It reveals the appearance of a limitation of the fluence "seen" by the sample surface, starting from the energy $E_{N L}^{a i r}$ and followed by a complete saturation for $E>E_{\text {ioniz. }}^{\text {air. }}$ (around $F_{0}^{\text {sample }} \sim 5 \mathrm{~J} / \mathrm{cm}^{2}$ in our experimental conditions). Therefore, for incident energy above $E_{\text {ioniz. }}^{a i r}$ the real fluence delivered on the target is strongly reduced compared to the fluence $F_{0}=2 E / \pi w_{0}^{2}$ calculated without nonlinear effects in air (i.e., considering that the beam is still Gaussian-shaped with $w_{0}$ size), therefore markedly modifying the ablation result that could be expected. We want to stress this particular point, since omitting to take it into account would yield strong misleading information when interpreting ablation experiments with few-cycle pulses in air.

Nevertheless, even if the beam is distorted (high energy case), the crater quality is maintained as shown by confocal microscopy (see 1D-profiles in Fig. 2). Surprisingly, high quality ablation with strongly distorted beams is therefore still possible. Moreover, thanks to the accurate beam characterization and proper fluence calibration, the crater diameter can even be predicted. Indeed, as we know the threshold peak fluence $F_{t h}$, it is straightforward to extract the beam diameter at local fluence equal to $F_{t h}$ (see Figure 3). As illustrated in Table I, the matching with measured crater diameters is excellent.

We also point out that at high energy, the beam undergoes spectral and temporal distortions, as it was measured in Ref. 12, leading, respectively, to enlargement and blue shift of the spectrum (due to air ionization) and a slight reduction of the pulse duration taking place in the central zone of the beam. However, spectral and temporal reshaping of the beam does not impede the prediction of the ablated crater based on the calibration of the fluence as well as its regularity and overall quality as shown in Fig. 2.
In conclusion, we have investigated if working in air may hamper surface ablation of dielectric materials by a tightly focused few-cycle pulse. We analyzed the transition from linear- to nonlinear- focusing regimes, including air ionization, therefore identifying three working ranges limited by $E_{N L}^{a i r}$ and $E_{\text {ioniz. }}^{\text {air }}$. The first important conclusion is that a fluence range exists $\left(F_{t h}<F<2.3 F_{t h}\right)$ for which propagation is free of any nonlinear effects, thus permitting to benefit from the advantages of few-cycle pulses for ablation. Second, upon increase of energy, we demonstrated by accurate characterization and proper calibration of $3 \mathrm{D}$ fluence distribution that the actual fluence delivered on the target surface is strongly limited, due to severe reshaping of the beam (including filamentation). Nevertheless, high-quality and fully predictable ablation is still reached. This work therefore provides useful information to the laser user (including also the possibility to translate the sample at the nonlinear focus location) to extend the capabilities of fewcycle pulses in a large range of operating fluence, far beyond the natural limit imposed by air ambiance.

The financial support of European Community, Ministry of Research and High Education, Region Provence-AlpesCôte d'Azur, Department of Bouches-du-Rhône, City of Marseille, CNRS, Aix-Marseille University, and Competitive Cluster Optitec, is gratefully acknowledged. We thank our colleague D. Grojo (LP3) for valuable discussions.

${ }^{1}$ W. Kautek, J. Krüger, M. Lenzner, S. Sartania, C. Spielmann, and F. Krausz, Appl. Phys. Lett. 69, 3146 (1996).

${ }^{2}$ M. Lenzner, J. Krüger, W. Kautek, and F. Krausz, Appl. Phys. A 68, 369 (1999).

${ }^{3}$ N. Sanner, O. Utéza, B. Chimier, M. Sentis, P. Lassonde, F. Légaré, and J. C. Kieffer, Appl. Phys. Lett. 96, 071111 (2010).

${ }^{4}$ B. Chimier, O. Utéza, N. Sanner, M. Sentis, T. Itina, P. Lassonde, F. Légaré, F. Vidal, and J. C. Kieffer, Phys. Rev. B 84, 094104 (2011).

${ }^{5}$ A. Couairon and A. Mysyrowicz, Phys. Rep. 441, 47 (2007).

${ }^{6}$ J. H. Marburger, Prog. Quantum Electron. 4, 35 (1975).

${ }^{7}$ O. G. Kosareva, W. Liu, N. A. Panov, J. Bernhardt, Z. Ji, M. Sharifi, R. Li, Z. Xu, J. Liu, Z. Wang, J. Ju, X. Lu, Y. Jiang, Y. Leng, X. Liang, V. P. Kandidov, and S. L. Chin, Laser Phys. 19, 1776 (2009).

${ }^{8}$ Y. T. Li, T. T. Xi, Z. Q. Hao, Z. Zhang, X. Y. Peng, K. Li, Z. Jin, Z. Y. Zheng, Q. Z. Yu, X. Lu, and J. Zhang, Opt. Express 15, 17973 (2007).

${ }^{9}$ D. G. Papazoglou and S. Tzortzakis, Appl. Phys. Lett. 93, 041120 (2008).

${ }^{10}$ P. P. Kiran, S. Bagchi, S. R. Krishnan, C. L. Arnold, G. R. Kumar, and A. Couairon, Phys. Rev. A 82, 013805 (2010).

${ }^{11}$ X.-L. Liu, X. Lu, X. Liu, T.-T. Xi, F. Liu, J.-L. Ma, and J. Zhang, Opt. Express 18, 26007 (2010).

${ }^{12}$ C. Pasquier, P. Blandin, R. Clady, N. Sanner, M. Sentis, O. Utéza, Y. Li, and S. Y. Long, Opt. Commun. 355, 230 (2015).

${ }^{13}$ J. M. Liu, Opt. Lett. 7, 196 (1982).

${ }^{14}$ S. L. Chin, Y. Chen, O. Kosareva, V. P. Kandidov, and F. Théberge, Laser Phys. 18, 962 (2008).

${ }^{15}$ S. L. Chin, Femtosecond Laser Filamentation, Springer Series on Atomic, Optical, and Plasma Physics, Vol. 55 (Springer, New York, 2010).

${ }^{16}$ M. Mlejnek, M. Kolesik, J. V. Moloney, and E. M. Wright, Phys. Rev. Lett. 83, 2938 (1999).

${ }^{17}$ W. Liu, F. Théberge, E. Arévalo, J.-F. Gravel, A. Becker, and S. L. Chin, Opt. Lett. 30, 2602 (2005).

${ }^{18}$ F. Courvoisier, V. Boutou, J. Kasparian, E. Salmon, G. Méjean, J. Yu, and J.-P. Wolf, Appl. Phys. Lett. 83, 213 (2003).

${ }^{19}$ A. Dubietis, E. Gaižauskas, G. Tamošauskas, and P. D. Trapani, Phys. Rev. Lett. 92, 253903 (2004).

${ }^{20}$ Y. E. Geints and A. A. Zemlyanov, Appl. Phys. B 101, 735 (2010).

${ }^{21}$ W. Hu, Y. C. Shin, and G. King, Appl. Phys. Lett. 99, 234104 (2011). 